\title{
A 2.5-D Diffraction Tomography Inversion Scheme for Ground Penetrating Radar
}

\author{
Meincke, Peter
}

Published in:

IEEE Antennas and Propagation Society International Symposium, 1999.

Link to article, DOI:

10.1109/APS.1999.788383

Publication date:

1999

Document Version

Publisher's PDF, also known as Version of record

Link back to DTU Orbit

Citation (APA):

Meincke, P. (1999). A 2.5-D Diffraction Tomography Inversion Scheme for Ground Penetrating Radar. In IEEE Antennas and Propagation Society International Symposium, 1999. (pp. 2132-2135). IEEE.

https://doi.org/10.1109/APS.1999.788383

\section{General rights}

Copyright and moral rights for the publications made accessible in the public portal are retained by the authors and/or other copyright owners and it is a condition of accessing publications that users recognise and abide by the legal requirements associated with these rights.

- Users may download and print one copy of any publication from the public portal for the purpose of private study or research.

- You may not further distribute the material or use it for any profit-making activity or commercial gain

- You may freely distribute the URL identifying the publication in the public portal

If you believe that this document breaches copyright please contact us providing details, and we will remove access to the work immediately and investigate your claim. 


\title{
A 2.5-D Diffraction Tomography Inversion Scheme for Ground Penetrating Radar
}

Peter M. Johansen'

Department of Electromagnetic Systems

Technical University of Denmark, DK-2800 Lyngby, Denmark

\begin{abstract}
A new 2.5-D inversion scheme is derived for ground penetrating radar (GPR) that applies to a monostatic fixed-offset measurement configuration. The inversion scheme, which is based upon the first Born approximation and the pseudo-inverse operator, takes rigorously into account the planar air-soil interface, the loss in the soil, and the characteristics of the antennas.
\end{abstract}

1. Introduction

Several inversion schemes, based upon the first Bom approximation and the concept of diffraction tomography (DT) [1], have been derived for monostatic ground penetrating radar (GPR) configurations [2-5]. In practical situations the GPR is usually situated upon a planar interface that separates air from soil and the soil is usually lossy. Therefore, it is important - as illustrated in [5] - to incorporate in the inversion the presence of the planar interface and the fact that the soil has loss. However, the inversion schemes in $[2,5]$ do not take into accoun the loss in the soil and only the one presented in [5] includes the planar air-soil interface. The present paper derives, to the knowledge of the author, the firs DT inversion scheme that accounts for both the planar interface and the lossy soil. The starting point is the forward model of [5] which is based upon the first Born approximation, the dyadic Green function for the planar air-soil interface, and an asymptotic approximation valid when the object is located deep (a few wavelengths) in the soil. However, instead of inverting this forward model using the inverse Fourier transform, as done in [5] - and thus neglecting loss in the soil - the pseudo-inverse operator of $[3,4]$ is used.

2. The Forwand Model

The GPR configuration is shown in Figure 1 in which a planar interface separates air from soil. A Cartesian $x y z$ coordinate system is introduced such that the $x y$ plane coincides with the interface and such that $z>0$ is air. An object, which is assumed infinitely long in the $\hat{x}$ direction, is buried in the soil. The propagation constant of air is $k_{0}(\omega)=\omega \sqrt{\mu_{0} \epsilon_{0}}$ and that of soil is $k_{1}(\omega)=\omega \sqrt{\mu_{0}\left(\epsilon_{1}+i \sigma_{1} / \omega\right)}$ (time factor $\exp (-i \omega t)$ ). The position of the receiving antenna is described by $\mathbf{r}_{r}=\mathbf{R}_{r}+\hat{\mathbf{z}} z_{r}$ and that of the transmitting antenna is $\mathbf{r}_{t}=\mathbf{r}_{r}+\mathbf{r}_{\Delta}$ with the fixed offset $\mathbf{r}_{\Delta}=\mathbf{R}_{\Delta}+\hat{\mathbf{z}} z_{\Delta}$. It is possible to derive a forward model that holds for arbitrary antennas [5]. However, for the sake of simplicity, it is here assumed that the antennas can be accurately modeled by Hertzian dipoles. It is also assumed that the contrast in conductivity $\Delta \sigma(y, z)=\sigma(y, z)-\sigma_{1}$ is much less than the contrast in permittivity, i.e., $\Delta \sigma(y, z) \ll \omega \Delta \varepsilon(y, z)$ over the frequency band of interest $\omega_{\min }<\omega<\omega_{\max }$. Then $\Delta \epsilon(y, z)$ can be related to the output of

'The Danish Technical Research Council is acknowledged for supporting this work. 


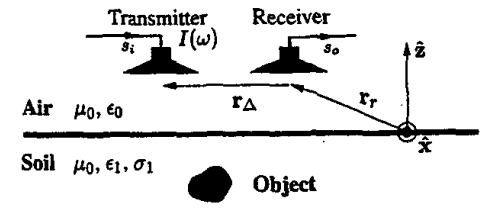

Figure 1: The fixed-offset monostatic GPR configuration.

the receiving antenna $s_{0}$ as $[5,(11)]$

$$
\begin{aligned}
\tilde{s}_{o}\left(k_{y}, z_{r}, \omega\right) & =\left(L \Delta \epsilon_{1}\right)\left(k_{y}, z_{r}, \omega\right)=-i \omega D\left(k_{y}, z_{r}, \omega\right) \int_{-\infty}^{\infty} d y^{\prime} \int_{-\infty}^{0} d z^{\prime} \\
& \cdot \exp \left(-i k_{y} y^{\prime}\right) \exp \left(-i \sqrt{\left.4 k_{1}^{2}(\omega)-\left|k_{y}\right|^{2} z^{\prime}\right) \Delta \epsilon_{1}\left(y^{\prime}, z^{\prime}\right)}\right.
\end{aligned}
$$

where $\Delta \epsilon_{1}=\Delta \epsilon / z^{\prime}$ and the Fourier transform $\tilde{s}_{o}$ of the output of the receiving antenna is obtained from $\tilde{s}_{o}\left(k_{y}, z_{\tau}, \omega\right)=\int_{-\infty}^{\infty} d y_{r} s_{o}\left(y_{r}, z_{r}, \omega\right) \exp \left(-i k_{y} y_{r}\right)$. The linear operator $L: U \rightarrow V$ maps the space $U$ onto the space $V . U$ is the space of square integrable functions of position $\left(y^{\prime}, z^{\prime}\right)$ confined within $z^{\prime}<0$.

$V$ is the space of square integrable functions defined on $\left\{\left(k_{y}, \omega\right) \mid \omega_{\min }<\omega<\right.$ $\left.\omega_{\max } \Lambda\left|k_{y}\right|<2 \operatorname{Re} k_{1}(\omega)\right\}$. Since $\tilde{s}_{0}=0$ for $\left|k_{y}\right|>2 \operatorname{Re} k_{1}(\omega)$ no evanescent plane waves in the soil are considered. Moreover, when the Hertzian dipoles are $\hat{\mathrm{x}}$-directed and have the same $x$ and $z$ coordinates $\left(x_{\Delta}=z_{\Delta}=0\right)$, the function $D$ in (1) is

$$
\begin{aligned}
D\left(k_{y}, z_{\tau}, \omega\right) & =\frac{i \omega^{2} \mu_{0}^{2} I(\omega)\left(4 k_{1}^{2}(\omega)-k_{y}^{2}\right)}{4 \pi k_{1}(\omega)\left(\sqrt{4 k_{0}^{2}(\omega)-k_{y}^{2}}+\sqrt{4 k_{1}^{2}(\omega)-k_{y}^{2}}\right)^{2}} \\
& \cdot \exp \left(i \cdot\left[\sqrt{4 k_{0}^{2}(\omega)-k_{y}^{2}} z_{\tau}+\frac{1}{2} k_{y} y_{\Delta}\right]\right)
\end{aligned}
$$

where $I(\omega)$ is the impressed current of the transmitting Hertzian dipole.

3. Inversion

The forward model (1) is now inverted using the (Tikhonov-regularized) pseudoinverse operator $[6, p .88]$

$$
\Delta \epsilon=z^{\prime} L^{\dagger}\left(L L^{\dagger}+\lambda^{2} I\right)^{-1} \tilde{s}_{0}
$$

where the adjoint operator $L^{\dagger}$, defined by $\left.<\tilde{s}_{0}, L \Delta \epsilon_{1}\right\rangle_{V}=\left\langle L^{\dagger} \hat{s}_{0}, \Delta \epsilon_{1}\right\rangle_{U}$ with the usual definition of the inner products, is

$$
\begin{aligned}
& \left(L^{\dagger} \tilde{s}_{o}\right)(y, z)=\int_{\omega_{\min }}^{\omega_{\max }} d \omega i \omega \int_{\left|k_{y}\right|<2 \operatorname{Rek}_{1}(\omega)} d k_{y} D^{*}\left(k_{y}, z_{T}, \omega\right) \\
& \cdot \exp \left(i k_{y} y\right) \exp \left(i \sqrt{4 k_{1}^{2}(\omega)-k_{y}^{2}} z\right) \tilde{s}_{o}\left(k_{y}, z_{\tau}, \omega\right) .
\end{aligned}
$$


Herein, * denotes the complex conjugate. The quantity $\lambda$ in (3) is a regularization parameter. However, in the numerical example in Section 4 a situation is considered which does not require regularization, i.e., $\lambda=0$. Now, the filtered data $\hat{s}_{0}^{f}$ is introduced as the solution to

$$
\left(L L^{\dagger}+\lambda^{2} I\right) \tilde{s}_{o}^{f}=\tilde{s}_{o} .
$$

Using this definition along with (3), the contrast in permittivity is obtained from

$$
\Delta \epsilon=z^{\prime} L^{\dagger} \tilde{s}_{0}^{f} \text {. }
$$

Hence, by solving (3) using the solution steps (5) and (6), the data are first filtered and then backpropagated to obtain the sought-for function $\Delta \epsilon$. Observe that the term $L L^{\dagger}$ in the filtering step (5) can be explicitly expressed as

$$
\begin{aligned}
\left(L L^{\dagger} \tilde{s}_{o}^{f}\right)\left(k_{y}, \omega\right) & =2 \pi i \omega D\left(k_{y}, z_{r}, \omega\right) \int_{\max \left(\omega_{\min }, \omega_{m}\left(k_{y}\right)\right)}^{\omega_{\text {max }}} d \omega^{\prime} \omega^{\prime} \\
& \cdot \frac{D^{*}\left(k_{y}, z_{r}, \omega^{\prime}\right) \tilde{s}_{o}^{f}\left(k_{y}, \omega^{\prime}\right)}{\sqrt{4 k_{1}^{2}(\omega)-\left|k_{y}\right|^{2}}-\sqrt{4 k_{1}^{2}\left(\omega^{\prime}\right)-\left|k_{y}\right|^{2}}}
\end{aligned}
$$

where $\omega_{m}\left(k_{y}\right)$ satisfies the relation $2 \operatorname{Re} k_{1}\left(\omega_{m}\left(k_{y}\right)\right)=\left|k_{y}\right|$. Hence, when inserting (7) into (5), an integral equation is obtained for determination of the filtered data $\tilde{s}_{0}^{f}$ for each value of $k_{y}$. This integral equation is solved numerically using pulse expansion and point matching. To this end, assume that the radar data $\tilde{s}_{o}$ is available at the equidistant frequencies $\omega_{p}=(p-1) \Delta \omega+\omega_{\operatorname{mim}}$, $\Delta \omega=\left(\omega_{\max }-\omega_{\min }\right) /\left(N_{\omega}-1\right), p=1, \ldots, N_{\omega}$. With $q\left(k_{y}\right)$ being the lowest positive integer satisfying $\omega_{m}\left(k_{y}\right)<q\left(k_{y}\right)$, the filtered data is expanded as $\tilde{s}_{o}^{f}\left(k_{y}, \omega\right)=\sum_{p=q\left(k_{y}\right)}^{N_{\omega}} s_{o p}^{f}\left(k_{y}\right) u_{p}(\omega)$ where the first $q\left(k_{y}\right)-1$ values of $s_{o p}^{f}\left(k_{y}\right)$ equal zero. The pulse expansion functions $u_{p}(\omega)$ are

$$
u_{p}(\omega)= \begin{cases}1 & \text { for } \omega_{\min }<\omega<\omega_{\min }+\Delta \omega / 2 \text { and } p=1 \\ \text { or for } \omega_{\max }-\Delta \omega / 2<\omega<\omega_{\max } \text { and } p=N_{\omega} \\ \text { or for }\left|\omega-\omega_{p}\right|<\Delta \omega / 2 \text { and } 1<p<N_{\omega}\end{cases}
$$

The resulting matrix equation for obtaining $\tilde{s}_{o p}^{f}\left(k_{y}\right)$ is easily derived by inserting the expansion for $\tilde{s}_{0}^{f}\left(k_{y}, \omega\right)$ into (7) and using (5) with point matching.

4. Numerical Example

The inversion scheme of Section 3 is now tested on synthetic GPR data. Figure 2 shows a dielectric pipe with a diameter of $15 \mathrm{~cm}$ and electromagnetic properties $\left(\epsilon_{\text {pipe }}, \sigma_{\text {pipe }}\right)=\left(8.1 \epsilon_{0}, 0.01 \mathrm{~S} / \mathrm{m}\right)$ located $1 \mathrm{~m}$ below the interface. The soil has $\left(\epsilon_{1}, \sigma_{1}\right)=\left(8 \epsilon_{0}, 0.01 \mathrm{~S} / \mathrm{m}\right)$. The synthetic GPR data is calculated from an eigenfunction expansion. It is assumed that the radar uses 60 frequencies equally spaced in the range $20 \mathrm{MHz}<f<1.3 \mathrm{GHz}$. Figure 3 shows the image of $\Delta \epsilon(y, z) / \epsilon_{0}$ obtained from (5) with $\lambda=0$ and from (6). The image is of high quality and it approximates well the correct value of $\Delta \epsilon=0.1 \epsilon_{0}$.

Acknowledgment

The author thanks Dr. T. B. Hansen from Schlumberger-Doll Research for providing the synthetic data used in the numerical example. 


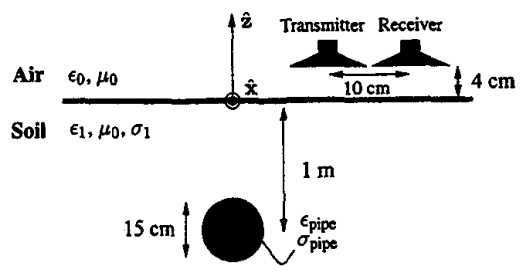

Figure 2: The configuration involving a pipe with $\Delta \epsilon=0.1 \epsilon_{0}$.

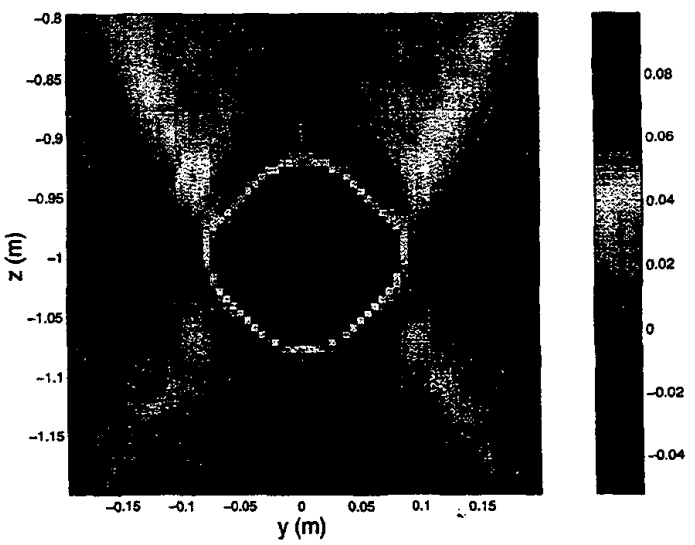

Figure 3: The image of $\Delta \epsilon(y, z) / \epsilon_{0}$.

References

[1] A. Devaney, "A filtered backpropagation algorithm for diffraction tomography," Ultrasonic Imaging, no. 4, pp. 336-350, 1982.

[2] J. Molyneux and A. Witten, "Diffraction tomographic imaging in a monostatic measurement geometry," IEEE Trans. Geosci. Remote Sensing, vol. 31,

pp. 507-511, Mar, 1993.
[3] R. Deming and A. Devaney, "A filtered backpropagation algorithm for GPR," J. Environmental and Eng. Geophysics, pp. 113-123, Jan. 1996.

[4] R. Deming and A. Devaney, "Diffraction tomography for multi-monostatic ground penetrating radar imaging," Inverse Problems, pp. 29-45, Feb. 1997.

[5] T. Hansen and P. Johansen, "Inversion scheme for ground penetrating radar that takes into account the planar air-soil interface." Submitted to IEEE Trans. Geosci. Remote Sensing.

[6] F. Natterer, The Mathematics of Computerized Tomography, John Wiley \& Sons, 1986. 